\title{
Evolution of Oxide Structures in Friction Stir Welded Alloy MA956
}

\author{
L.N. Brewer ${ }^{1,2}$, B.W. Baker ${ }^{1,3}$, M.J. Bird ${ }^{1}$, and S. Osswald, ${ }^{1,4}$
}

1. Mechanical and Aerospace Engineering, Naval Postgraduate School, Monterey, CA, USA

2. Metallurgical and Materials Engineering, University of Alabama, Tuscaloosa, AL, USA

3. Mechanical Engineering Department, United States Naval Academy, Annapolis, MD, USA

4. Purdue University, West Lafayette, IN, USA

Oxide dispersion strengthened alloys are of great potential importance for advanced fission and fusion reactors because of their high temperature strength, creep resistance, and radiation damage resistance.[1] Unfortunately, these materials are not readily joined by fusion welding as the dispersed oxides agglomerate and coarsen in the weld pool, thus drastically reducing the strength of the welded material. Friction stir welding (FSW) can successfully join these materials through a solid-state bonding process, but there is still some reduction in strength due to evolution of the oxide structure.[2] This paper examines the changes in oxide size and phase as a function of FSW parameters in the alloy MA956.

Two plates of MA956 (Fe-19.9Cr-4.8Al-0.39Ti- $0.51 \mathrm{Y}_{2} \mathrm{O}_{3}-0.023 \mathrm{C}$ ) were friction stir welded over a series of tool rotation and translation speeds, from 200-500 RPM and from 25-175 millimeters per minute (MMPM). Cross-sectional samples for electron microscopy, Raman spectroscopy, and x-ray microanalysis were prepared by cutting the plate perpendicular to the weld and using metallographic techniques down to a finish of 0.05 um colloidal silica. SEM and EDX analyses were completed using a Zeiss Neon 40 scanning electron microscope at $10-12 \mathrm{keV}$ beam voltage, $60 \mu \mathrm{m}$ objective aperture, and a probe current of $1 \mathrm{nA}$. Both EDX maps and point spectra were collected using the EDAX Genesis system and a silicon drift detector. Raman spectroscopy measurements were performed with Renishaw's inVia Raman microspectrometer using 514 and $785 \mathrm{~nm}$ laser excitation wavelengths.

SEM imaging and $\mathrm{x}$-ray mapping showed a clear increase in oxide particle size in the stir zone of welds with large heat input, i.e. low tool translation speed and high rotation speeds. The yttrium-based oxides in the base material are approximately $10 \mathrm{~nm}$ is size as determined by SAXS and STEM measurements. After FSW, yttrium-aluminum oxide particles as large as $350 \mathrm{~nm}$ in size were observed. Interestingly, the spacing between coarsened oxides was approximately $3 \mu \mathrm{m}$ for all of the high heat input conditions, suggesting that the oxide coarsening is not just from Ostwald ripening alone.

The Raman data shows that the phase of the oxides is also changing as the oxides coarsen. The highest heat input conditions (e.g. 500RPM/25 MMPM) have a majority of yttrium aluminum garnet (YAG) particles with some yttrium aluminum perovskite (YAP) particles present in the stir zone. Lower heat input conditions, e.g. $400 \mathrm{RPM} / 100 \mathrm{MMPM}$, do not show signal from the YAG phase but instead primarily from the YAP phase. These results are in agreement with the work of Chen et al., [3] but the present results are the first to show the direct connection between the FSW conditions and the size and phase evolution of the oxides. These results are important because they underscore the need to control oxide phase stability during FSW in order to maintain the strength and radiation damage resistance of the welded material.

\section{References:}

[1] P. Andresen, F.A. Garner, et al., EPRI, Palo Alto, CA, 2012, pp. 1-320.

[2] B.W. Baker, T.R. McNelley, et al., Materials Science and Engineering-A, 589 (2014) 217-227.

[3] C.L. Chen, P. Wang, et al., Materials at High Temperatures, 26 (2009) 299-303. 

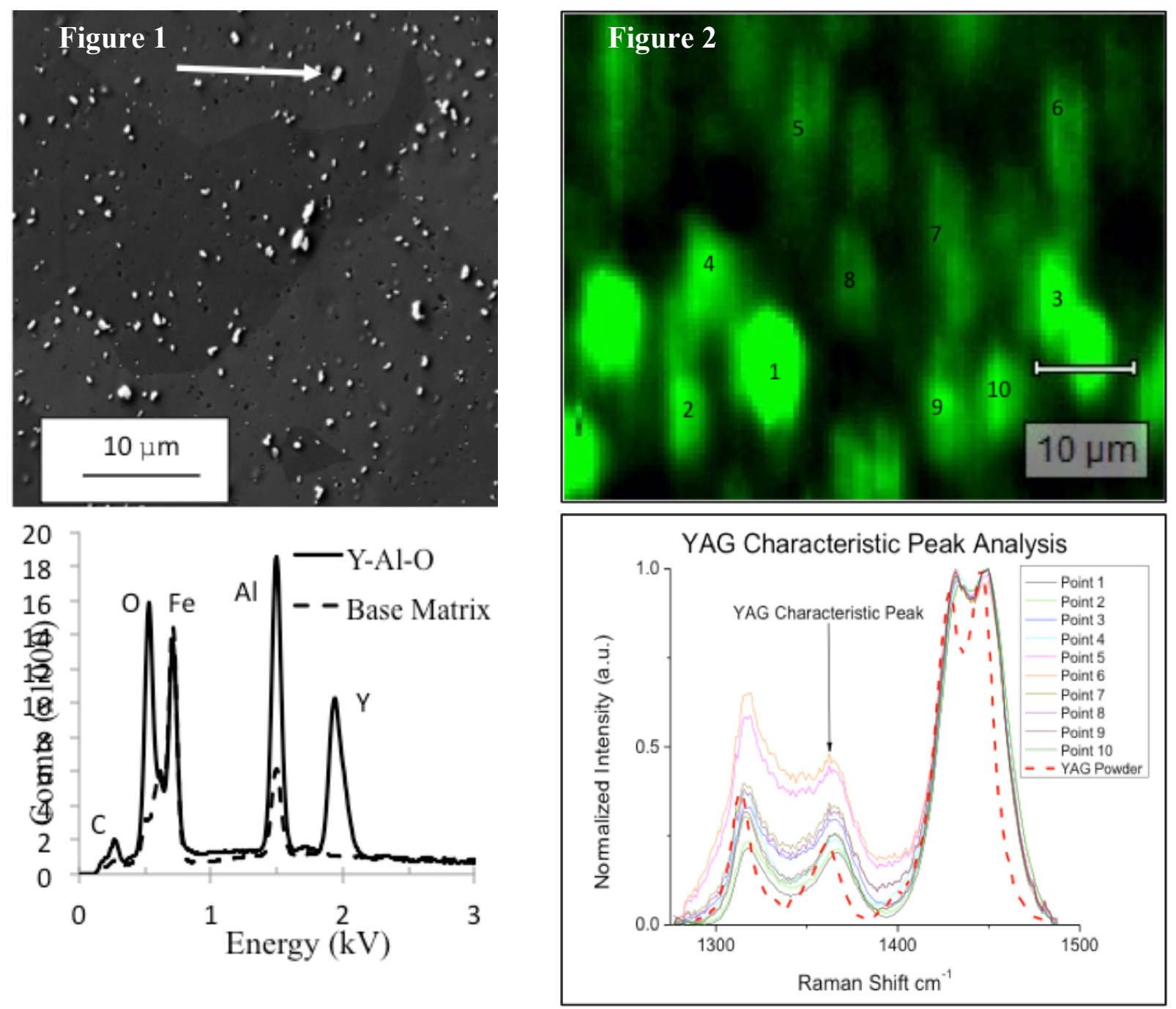

Figure 1. Secondary electron image (top) of oxides from friction stir weld at high heat input condition (500 RPM/25 MMPM). The white arrow indicates an individual, coarsened oxide (Y-Al-O). (bottom) EDX spectra showing signals from the matrix and the oxide particle.

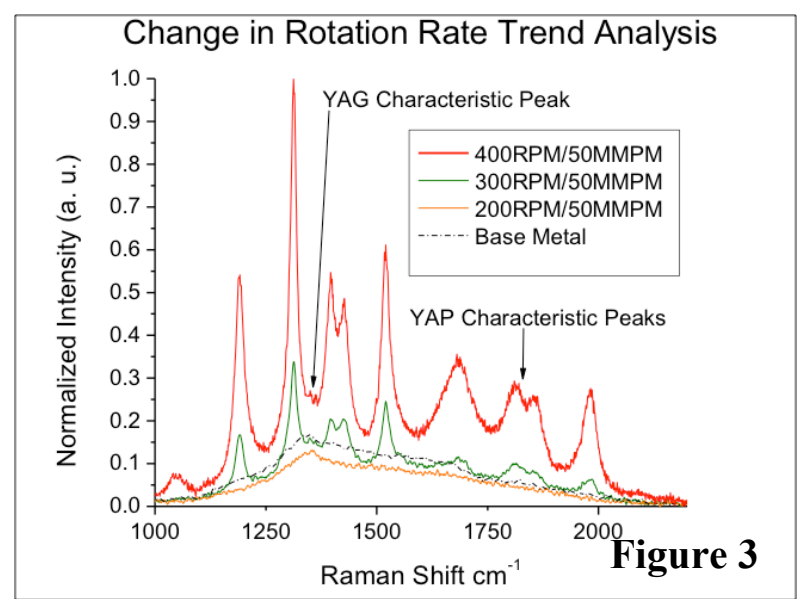

Figure 2. Raman spectral map (top) of particles with dominant features from the YAG phase. The individual numbers correspond to spectra from individual particles. (bottom) The individual Raman spectra that correspond to the numbered oxide particles.

Figure 3. Systematic change in Raman spectra as a function of increasing FSW tool rotation speed. 